\title{
Managing Heart Failure in Long-Term Care: Recommendations from an Interprofessional Stakeholder Consultation*
}

\author{
George A. Heckman, ${ }^{1}$ Veronique M. Boscart, ${ }^{2}$ Teresa D'Elia, ${ }^{3}$ Mary Lou Kelley, ${ }^{4}$ \\ Sharon Kaasalainen, ${ }^{5}$ Carrie A. McAiney, ${ }^{6}$ Mary-Lou van der Horst, ${ }^{7}$ and Robert S. McKelvie ${ }^{8}$
}

\begin{abstract}
RÉSUMÉ
Insuffisance cardiaque (IC) affecte autant que 20 pour cent des résidents en soins de longue durée (SLD), et est associée à la morbidité substantielle, la mortalité et l'utilisation des services de santé. L'objectif de notre étude était de formuler des recommandations sur la mise en œuvre de processus pour prendre soin de l'insuffisance cardiaque dans SLD. Un processus de consultation itérative triphasé avec les parties prenantes a été guidé par la participation d'un panel d'experts et a servi à élaborer des recommandations. Dix-sept recommandations ont été faites. Éléments clés des celles-ci se concentrent sur l'amélioration de la communication interprofessionnelle et accroître les connaissances relatives à l'insuffisance cardiaque entre tous les intervenants dans SLD. Des recommandations systématiques incluent améliorer la communication entre les foyers de SLD et soins aigus et autres prestataires de santé externes, et développer des interventions dans l'ensemble des installations afin de réduire les apports de sodium alimentaire et d'augmenter l'activité physique.
\end{abstract}

\section{ABSTRACT}

Heart failure (HF) affects up to 20 per cent of residents in long-term care (LTC) and is associated with substantial morbidity, mortality, and health service utilization. Our study objective was to formulate recommendations on implementing HF care processes in LTC. A three-phase and iterative stakeholder consultation process, guided by expert panel input, was employed to develop recommendations on implementing care processes for HF in LTC. This article presents the results of the third phase, which consisted of a series of interdisciplinary workshops. We developed 17 recommendations. Key elements of these recommendations focus on improving interprofessional communication and improving HF-related knowledge among all LTC stakeholders. Engaging frontline staff, including personal support workers, was stated as an essential component of all recommendations. System-level recommendations include improving communication between LTC homes and acute care and other external health service providers, and developing facility-wide interventions to reduce dietary sodium intake and increase physical activity.

1 Schlegel-University of Waterloo Research Institute for Aging and School of Public Health and Health Systems, University of Waterloo

2 School of Health \& Life Sciences and Community Services, Conestoga College Institute of Technology and Advanced Learning, Kitchener

3 Institute for Work \& Health, Toronto

4 School of Social Work, Lakehead University, Thunder Bay

5 Faculty of Health Sciences, McMaster University, Ontario

6 Department of Psychiatry and Behavioural Neurosciences, McMaster University and Director of Research and Evaluation, Seniors Mental Health Service, St. Joseph's Healthcare Hamilton

7 Schlegel-University of Waterloo Research Institute for Aging, Waterloo

8 Department of Medicine, Western University, London, Ontario

* The authors would like to acknowledge the nursing homes and participants in this study. We are grateful for the administrative assistance of Nisreen Murad and Christie Gennings. We also acknowledge the contributions to the project of Catherine Demers, Ian Newhouse, Erin Tjam, Patricia Strachan, Paul Stolee, Jill Marcella, Alesha Gaudet. Jayanthini Nadarajah, Osman Osman, Amy Horton, Hellen Jarman, Sonja Habjan, Chase McMurren, and Amy Montour. GAH and VMB are grateful for salary support from the Schlegel-UW Research Institute for Aging Schlegel Research Chairs in Geriatric Medicine, and Schlegel Villages, Conestoga College, CIHR/Industrial Research Chair for Colleges in Seniors Care, respectively. This study was funded by a Grant-in-Aid from the Heart and Stroke Foundation of Ontario (HSFO; NA-6811). HSFO did not play a role in the design or conduct of the study, the collection, management, analysis, or interpretation of data, or preparation, review, or approval of the completed manuscript.

Canadian Journal on Aging / La Revue canadienne du vieillissement 35 (4) : 447-464 (2016)

(c) Canadian Association on Gerontology 2016. This is an Open Access article, distributed under the terms of the Creative

Commons Attribution licence (http://creativecommons.org/licenses/by/4.0/), which permits unrestricted re-use,

distribution, and reproduction in any medium, provided the original work is properly cited.

doi:10.1017/S071498081600043X 
Competing interests: None of the authors have any potential conflicts of interest to declare.

Manuscript received: / manuscrit reçu : 03/02/16

Manuscript accepted: / manuscrit accepté : 06/06/16

Mots clés : vieillissement, insuffisance cardiaque, soins à longue durée, fragilité, interprofessionnel, -lle, aidant, -te

Keywords: aging, heart failure, long-term care, frailty, interprofessional, health care aides

La correspondance et les demandes de tire-à-part doivent être adressées à : / Correspondence and requests for offprints should be sent to:

George A. Heckman, M.D.

Schlegel Research Chair in Geriatric Medicine, Associate Professor

School of Public Health and Health Systems

University of Waterloo, BMH 2304

200 University Avenue West

Waterloo, ON N2L 3G1

(ggheckma@uwaterloo.ca)

Heart failure (HF) is a chronic syndrome that develops when the heart can no longer sustain sufficient output to meet the metabolic demands of the body (McMurray et al., 2012). Worldwide, HF affects over 26 million individuals, and annual hospitalization rates in North America and Europe for a primary diagnosis of HF are counted in the millions (Ambrosy et al., 2014). The prevalence of HF is rising due to population aging, and the remaining lifetime risk for octogenarians approaches 20 per cent (Bleumink et al., 2004; Go et al., 2014). The burden of HF disproportionately affects older persons, in whom it is a leading cause of death and health service utilization (Heckman, Tannenbaum, Costa, Harkness, \& McKelvie, 2014). HF in older persons is further complicated by concurrent multimorbidity and geriatric syndromes, including frailty, cognitive impairment, and functional decline, and a sizeable proportion of seniors with HF are ultimately admitted into a long-term care (LTC) or nursing home (Heckman, Tannenbaum, et al., 2014).

LTC homes provide 24-hour nursing care and personal support to persons for whom continued residence in the community is no longer feasible because of multiple concurrent age-associated conditions and disabilities (Hirdes, Mitchell, Maxwell, \& White, 2011). The prevalence of HF in LTC is approximately 20 per cent, and the one-year mortality rate exceeds 50 per cent (Heckman, Boscart, \& McKelvie, 2014). HF accounts for a significant proportion of transfers of LTC residents to acute care, almost a third of which are readmissions (Heckman, Boscart, \& McKelvie, 2014). A recent population-based study in Ontario's LTC homes showed that HF accounted for 16 per cent of emergency department visits over a six-month period (Gruneir et al., 2010). The impact of emergency department visits by LTC residents is substantial and includes costly ambulance transport, long waits in emergency departments for inpatient beds, and hospital stays of up to a week that are often complicated by delirium and functional decline
(Heckman, Boscart, \& McKelvie, 2014). It has been suggested that many acute care visits could be prevented if appropriate HF care processes were in place in LTC (Gruneir et al., 2010).

International HF management guidelines endorse standard HF therapies for older patients (Hunt et al., 2009; McKelvie et al., 2013; McMurray et al., 2012). However, numerous studies have shown that current care processes for older patients with HF, particularly those in LTC, do not reflect guidelines (Heckman, Boscart, \& McKelvie, 2014). HF in LTC residents is often misdiagnosed, with infrequent monitoring of pertinent serum chemistry and therapeutic drug levels, and limited assessment of cardiac function with echocardiography (Heckman, Boscart, \& McKelvie, 2014). In addition, LTC residents are less likely than community-dwelling seniors to be prescribed first-line medications such as angiotensin converting enzyme inhibitors (ACEi) and beta-blockers (Heckman, Boscart, \& McKelvie, 2014). Although HF management programs for communitydwelling patients have been shown to reduce acute care use and mortality (Hunt et al., 2009; McKelvie et al., 2013; McMurray et al., 2012), comparable programs are uncommon in LTC. A small number of studies in Europe, the United States, and Canada have shown that guideline-based HF management programs in LTC may reduce hospitalization rates, though the generalizability of these programs is limited as they target relatively highly functioning LTC residents, benefit from close proximity of expert and diagnostic services, and fail to consider optimal interprofessional collaboration and sustainability (Boxer et al., 2012; Hancock et al., 2012; Jacobs, 2011; Martinen \& Freundl, 2004; Valle, Chinellato, Milani, \& di Piave, 2001; Tjam et al., 2006).

We conducted a mixed-methods study, carried out in three phases, in order to develop recommendations on how to implement feasible and effective care processes 
to manage HF in LTC. The results of each phase informed the subsequent phase (Figure 1). The first (Delphi survey) and second (focus group and individual interviews) phases focused on gathering LTC residents' and staff caregivers' (formal and informal) experiences of HF care and recognizing barriers and potential solutions to guideline-based HF management in LTC. The results of each phase were reviewed by an interprofessional panel of experts in cardiology; geriatrics; and primary, specialized, and long-term care. The panel also included physicians, nurses, and pharmacists (Table 1). The role of the expert panel was to ensure that findings from each phase remained consistent with HF guidelines, and consistent with the scope of each staff role in LTC. Findings from these phases are published elsewhere (Heckman, Boscart, McKelvie, et al., 2014; Kaasalainen et al., 2013; Marcella et al., 2012; Newhouse et al., 2012; Strachan et al., 2014). This article reports on the third and final phase of the study, the purpose of which was to formulate recommendations on developing and implementing HF care processes for LTC, based on the Canadian Cardiovascular Society HF guidelines, that optimally utilize the skill sets of all LTC staff roles, are minimally disruptive to work routines, and focus on achieving outcomes relevant to LTC residents.

\section{Previous Work and Background}

To develop and implement a feasible, effective, and sustainable HF management program in LTC, a staged and systematic approach is required. We completed the first two study phases in order to identify key barriers impeding optimal HF care in LTC, and thus inform the development of the recommendations for HF care processes in LTC. Findings are summarized below.

Although Phase 1 confirmed that knowledge deficits about HF exist among all staff, problematic interprofessional communication was recognized as a fundamental obstacle to optimizing HF care (Strachan et al., 2014).

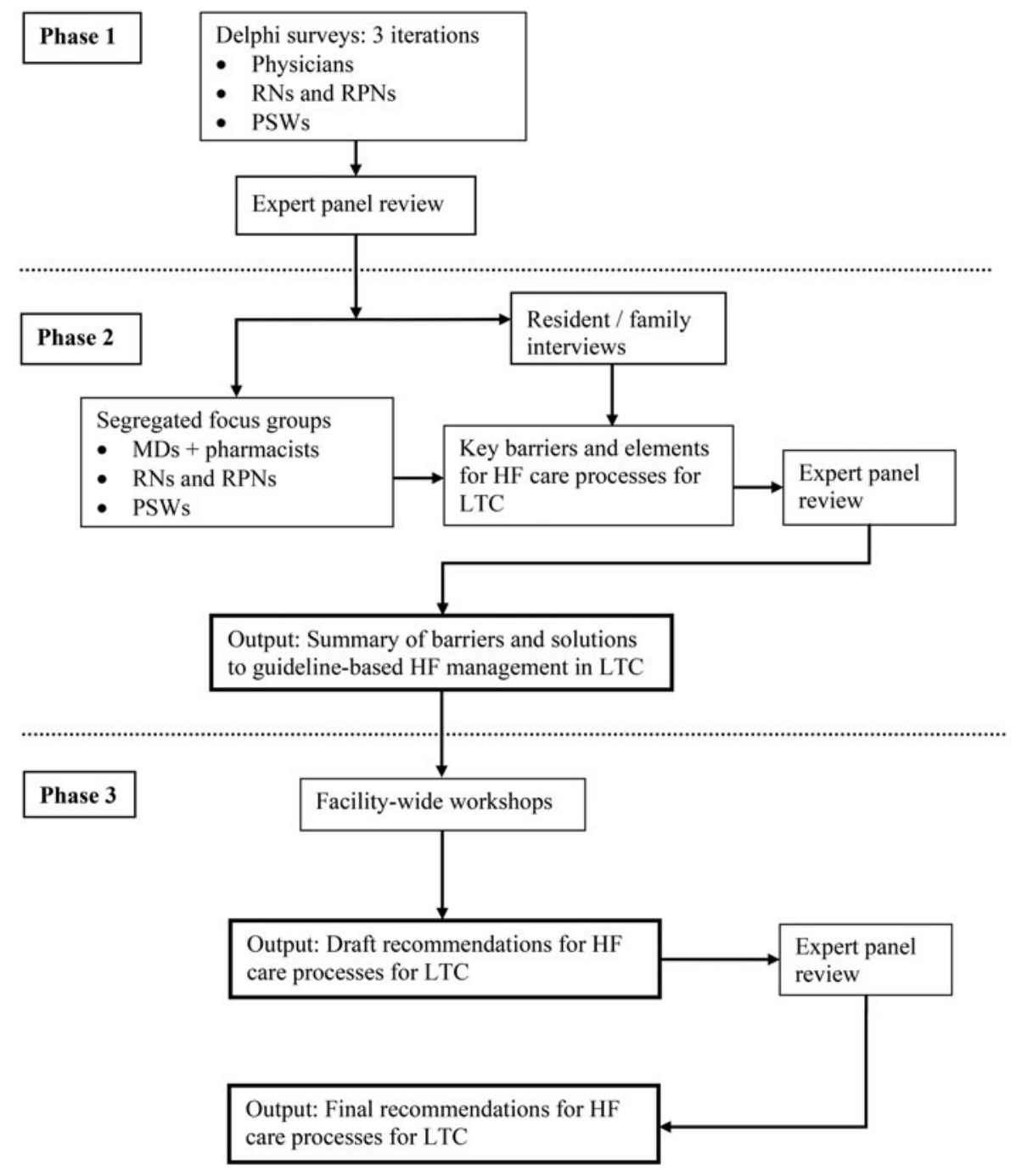

Figure 1: Flow diagram illustrating Heart Failure Care Process development activities 
Table 1: Study investigators and composition of the interdisciplinary expert panel

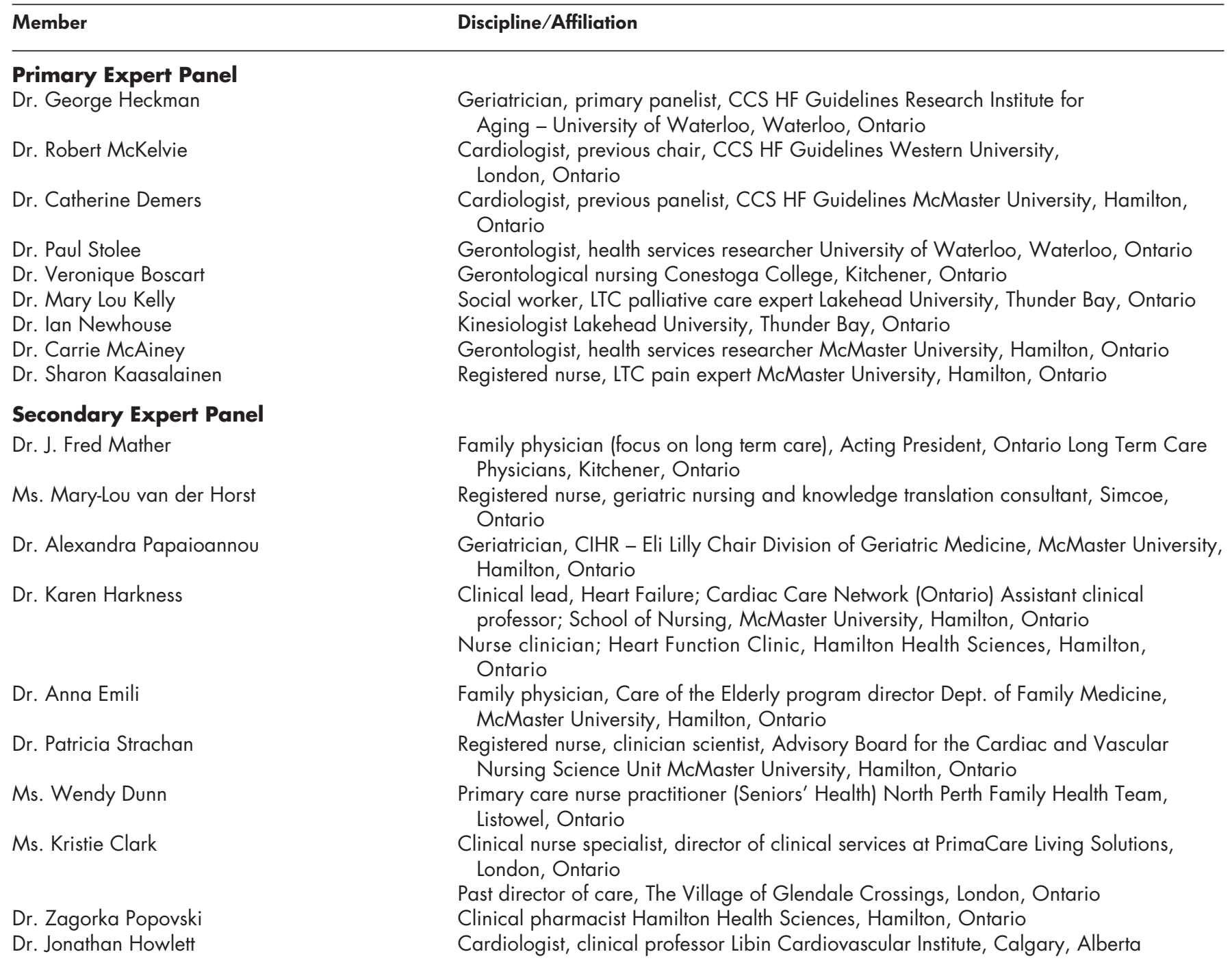

\section{CCS HF = Canadian Cardiovascular Society Heart Failure; LTC = long-term care}

We explored these factors in greater depth in Phase 2. Factors underlying interprofessional miscommunication included role confusion among team members, lack of mutual respect between staff groups, and mistrust in the abilities of others (Heckman, Boscart, McKelvie, et al., 2014; Marcella et al., 2012; Newhouse et al., 2012; Strachan et al., 2014). The factors were exacerbated by heavy workloads and a rigid, hierarchical approach to decision-making excluding personal support workers (PSWs, unregulated but certified caregivers with the most frequent resident contact), residents, and families (Heckman, Boscart, McKelvie, et al., 2014; Strachan et al., 2014). Staff shortages and insufficient funding have caused changes in roles among LTC staff, including the devolution of certain tasks and responsibilities from registered nurses (RNs) to less skilled registered practical nurses (RPNs). As a result, the Phase 1 study found that RNs spent much of their time on documentation and administration tasks rather than on resident care (Newhouse et al., 2012).

PSWs were deemed by all stakeholders to be best positioned to detect early signs of acute illness or exacerbations of chronic conditions such as HF, which often present with non-specific symptoms such as anorexia, or changes in cognition, mobility, or function (Heckman, Boscart, McKelvie, et al., 2014; Kaasalainen et al., 2013; Marcella et al., 2012; Newhouse et al., 2012; Strachan et al., 2014). These more subtle features of acute illness are often perceived as mundane in the LTC context, and thus dismissed. Differentiation between these symptoms requires the clinical assessment of experienced and knowledgeable RNs, but as RNs are removed from bedside care, symptoms of worsening of HF often go unnoticed (Newhouse et al., 2012). In addition, current LTC environments and staffing models are not always conducive for PSWs to report their observations on 
resident status or changing clinical presentation, and their input is at times dismissed, or PSWs are perceived as being insufficiently trained to systematically document or communicate these observations and/or changes (Heckman, Boscart, McKelvie, et al., 2014; Strachan et al., 2014).

Lastly, LTC residents with HF and their families in Phase 1 expressed a lack of understanding of their condition, and were uncertain about when and with whom to communicate their health concerns (Kaasalainen et al., 2013). Greater commitment from all LTC stakeholders, including administrators, was seen as essential to promote more effective interprofessional collaboration in LTC (Heckman, Boscart, McKelvie, et al., 2014; Kaasalainen et al., 2013; Marcella et al., 2012; Newhouse et al., 2012; Strachan et al., 2014).

Phase 2 also revealed significant gaps in clinical skills and knowledge about HF care among all LTC staff, and which are exacerbated by the medical complexity of LTC residents (Heckman, Boscart, McKelvie, et al., 2014; Marcella et al., 2012; Newhouse et al., 2012). Frontline staff reported insufficient training to confidently observe, assess, interpret, and report clinical changes or to implement and evaluate care plans and clinical interventions, whereas more highly skilled staff found themselves removed from the bedside, leading to clinical decisionmaking based on second-hand, poorly communicated, and thus unreliable information from others (Heckman, Boscart, McKelvie, et al., 2014; Marcella et al., 2012; Newhouse et al., 2012). In order to improve HF care for LTC residents, all staff identified a need to better understand basic physiology and clinical and management skills specific to frail LTC residents with HF, as well as how to apply this information to guide and sustain clinical processes. Multimodal interprofessional education including bedside teaching by expert clinicians and regular case conferences with the entire care team were seen as desirable and valuable learning opportunities (Marcella et al., 2012; Newhouse et al., 2012).

\section{Methods}

\section{Expert Panel Review of Phases 1 and 2}

Phase 1 and 2 findings were reviewed and discussed by the expert panel at a teleconference. The panel agreed that a staged approach to the development of HF care processes for LTC is required, starting with (1) interventions within the LTC home; and (2) interventions geared to the system level and broader community (Table 2).

The discussions of the expert panel informed the third and final phase of this study, the objectives of which were to develop specific recommendations on how to implement HF care processes in LTC. To address these objectives, we used an iterative consensus approach.
First, the findings of the expert panel were presented at interprofessional consultative workshops held in the three LTC homes in which prior study phases had taken place. Workshop attendees were then asked to elaborate in greater detail the practical aspects of implementing HF care processes for LTC. Workshop results were summarized and reviewed by the expert panel, which finalized the HF care process recommendations. Methods are described in more detail below.

\section{Consultative Workshops}

All staff (physicians, nurses, PSWs, and administrators) at participating LTC homes were invited to participate in the workshop, and an effort was made to have all staff roles represented in each session. We limited group sizes to no more than 10 participants. When the number of session participants exceeded 10, two smaller groups were created with proportional representation from different staff roles.

First, we presented the results of Phase 1 and 2 and the feedback from the expert panel to the study participant groups. Second, we engaged participants in a facilitated discussion, the goal of which was to expand upon the key considerations and elements identified by the expert panel, addressing these from three implementation perspectives: (1) What processes and resources are currently available in LTC? (2) What additional resources are required to optimally implement HF care processes in LTC? and (3) What steps and activities are required to successfully implement HF care processes in LTC?

\section{Development of Recommendations}

At each workshop, two research assistants independently took notes that were organized in a matrix containing the themes and elements outlined by the expert panel and the three implementation perspectives (Table 3). Information thus collected at each workshop was collated into a single master template, which the study investigators analysed using an inductive process to draft recommendations for the development of HF care processes for LTC. The expert panel was then asked to provide feedback on the recommendations, which were finalized based on this feedback.

Ethics clearance for all procedures was provided by the Research Ethics Boards of the University of Waterloo, McMaster University, and Lakehead University.

\section{Results \\ Workshops}

A total of 30 participants, representing a variety of care professions involved in LTC, participated in five 
Table 2: Summary of expert panel review of Phases 1 and 2

\begin{tabular}{ll}
\hline Broad Themes & Key Considerations \\
\hline Interventions within the LTC home & $\begin{array}{l}\text { - Developing processes to improve IP communication } \\
\text { - Developing educational interventions tailored to different levels of } \\
\text { staff and that focus on: } \\
\text {-understanding and recognizing HF in older persons } \\
\text {-diagnosis of and managing HF (includes tracking weights and } \\
\text { avoiding sodium) }\end{array}$ \\
$\begin{array}{l}\text {-appropriate prescribing } \\
\text {-advanced care planning }\end{array}$ \\
$\begin{array}{c}\text { Interventions geared to the system level and broader community } \\
\text { settings (e.g., hospitals, home care, clinics) } \\
\text { Develop policy-level strategies to reduce sodium intake and } \\
\text { promote physical activity in a variety of settings }\end{array}$
\end{tabular}

HF = heart failure; IP = interprofessional; LTC = long-term care

workshops (Table 4). The participants responded positively to the findings of Phases 1 and 2, and to the expert panel feedback. Staff recognized and acknowledged residents' and caregivers' experiences of HF care, as well as barriers and potential solutions to guideline-based HF management in LTC. The facilitated discussions led to the identification of several strategies to implement HF care processes.

Table 3: Workshop discussion matrix

Interventions within the Long-Term Care Home

Develop Processes to Improve HF Knowledge and Interprofessional Communication within the Home

Available Resources

Resources needed

Actions required

Monitoring Weights

Available Resources

Resources needed

Actions required

Appropriate Prescribing for Heart Failure

Available Resources

Resources needed

Actions required

Communication with Families and Advance Care Planning

Available Resources

Resources needed

Actions required

Interventions Geared to the System Level and Broader Community

External Communication: Hospitals/Other External Health Service Providers

Available Resources

Resources needed

Actions required

External Communication: Health informatics considerations

Available Resources

Resources needed

Actions required

Facility and System-Level Interventions to Ensure Heart-Failure-Appropriate Nutrition

Available Resources

Resources needed

Actions required

Support for Physical Activity Promotion

Available Resources

Resources needed

Actions required 
Table 4: Workshop Sites and Attendee Professions

\begin{tabular}{|c|c|}
\hline Site & Attendees \\
\hline Nursing home 2, rural southwestern Ontario (one workshop) & $\begin{array}{l}\text { Registered nurse }(n=2) \\
\text { Registered practical nurse }(n=2) \\
\text { Personal support workers }(n=2) \\
\text { Administration }(n=1)\end{array}$ \\
\hline
\end{tabular}

\section{Interventions within the LTC Home}

Study participants agreed on the most important elements of HF management in LTC - effective interprofessional communication and tailored educational interventions and that these are inextricably linked. Below, strategies identified by workshop participants regarding interprofessional communication and HF education are presented.

\section{Strategies to Improve Interprofessional Communication}

Participants agreed that effective communication is critical to ensure the timely sharing of accurate information about residents to allow all team members to contribute to the formulation of appropriate treatment recommendations. Processes to ensure effective and mutually respectful, reciprocal, and confident communication among staff are essential to improve all aspects of care. Several examples of strategies to address communication barriers were provided. Any or all of these strategies were felt to be easily adaptable to HF-specific information, including clinical observations, planned treatments, and follow-up instructions (Table 5).

Workshop attendees noted the importance of effective communication during specific care episodes, including shift change, unanticipated resident deterioration, physician rounds, weight monitoring, and new admissions to LTC.

Shift change and ad hoc communication in cases of resident deterioration: Effective communication at shift change was noted by workshop discussants as particularly crucial to ensure quality care, as it was at this juncture that failure to transmit critical information was most likely to occur. Another critical event is the identification by a PSW of a resident whose condition is deteriorating, as is the importance of ad hoc communication processes to interrupt normal work routines and ensure timely assessment. Participants believed a consistent approach to be essential, including formally mapped out and mandated communication protocols, and defining what constitutes "critical information", depending on individual LTC staff's knowledge and expertise. Table 6 lists a variety of methods that were shared on how to ensure that key information or "highlights" from previous shifts can be shared, as well as potential pitfalls.

Communication during physician rounds and resident care planning meetings: Participants reported that wide variability exists in communication patterns between physicians and the rest of the care team at their respective LTC homes. In extreme cases, a team discussion on resident issues occurs only at an annual care conference. Other LTC sites have weekly team rounds with the physician. Attendance at meetings is also variable. At some sites, annual meetings occur without some key members, and at times even the physician is absent. PSWs are often excluded. In other facilities, the entire team is present with the resident and a family representative.

A promising approach has the physician rounding regularly with nurses and PSWs, who are best positioned to report on resident habits and changes in condition, and who can provide valuable insight as to whether a treatment change has been effective. In general, if physician rounds follow a regular schedule, PSWs can participate constructively with minimal delays in their schedule. This approach was felt to promote greater trust among staff, facilitate effective communication, and facilitate teamwork. 
Table 5: Communication strategies that could be adapted to HF management

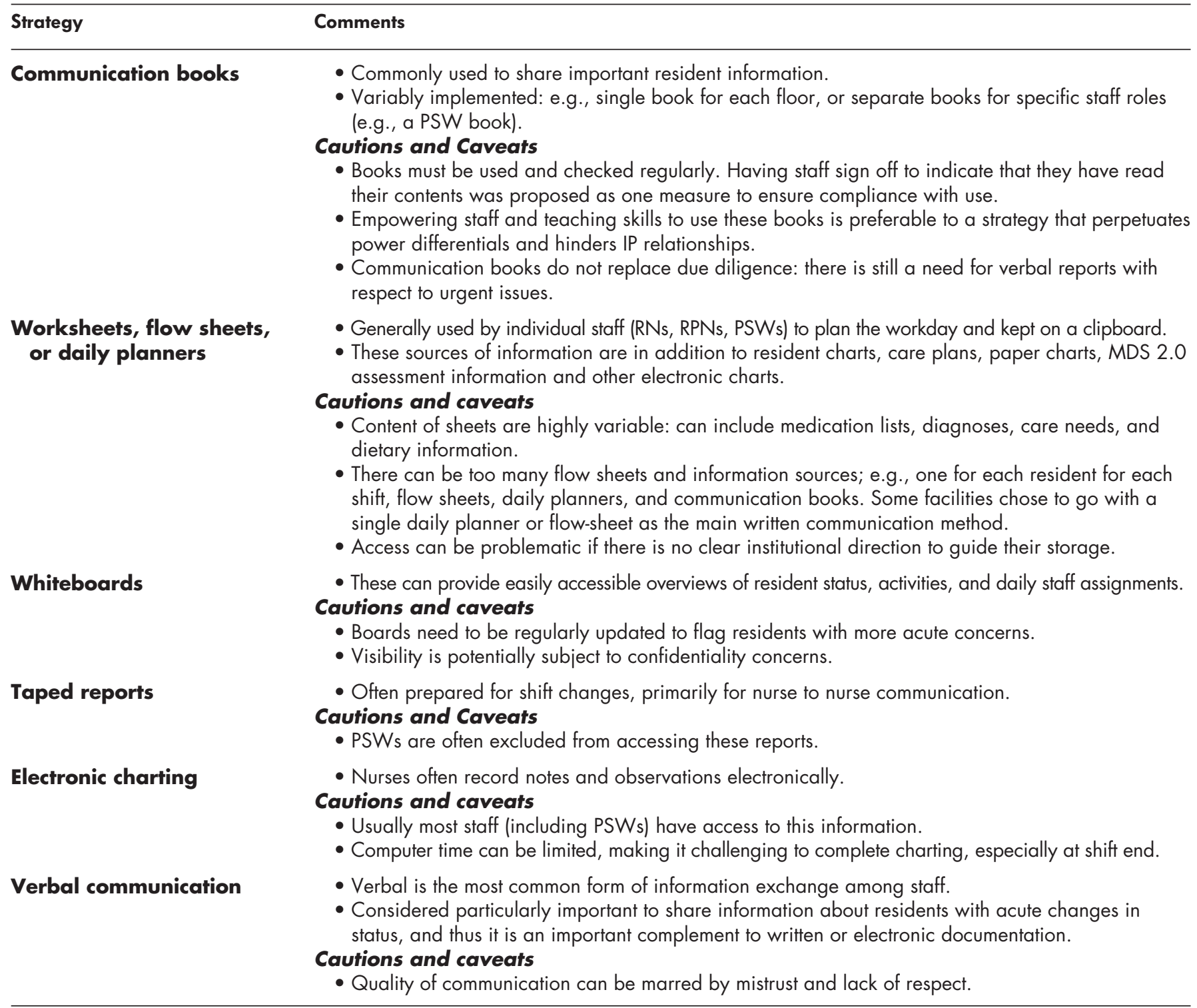

IP = interprofessional; PSW = personal support worker

Communication upon new admissions to LTC: Communication of health information for newly admitted LTC residents was also identified as an area of concern by workshop participants. Whereas Ontario, like many jurisdictions, mandates assessment of potential LTC residents with standardized instruments, this information is often incomplete upon arrival of a resident at a receiving facility. In cases when residents await LTC placement for extended periods of time, information is not fully updated before resident transfer despite often significant interim changes in clinical status. The quality of the information transferred may also depend on which home care case manager was involved with the resident awaiting LTC placement.

Past medical information may be more accessible when residents are transferred from one LTC home to another.
For residents admitted from the community, records from family physicians may be available, but generally this information is incomplete. Vaccination records in particular are difficult to obtain, as are consultation records from specialists seen in the community. Workshop participants generally agreed that integrated and standardized electronic records and assessment systems linking primary care, home care, pharmaceutical records, diagnostic testing, and hospital records are sorely needed.

Communication and regularly monitoring weights of residents with HF: All participants agreed that staff education was particularly crucial towards ensuring a successful weight monitoring strategy. The ordering of regular and frequent weighings was viewed, particularly by participating PSWs, as a significant imposition 
Table 6: Sharing key information about residents: methods and piffalls

Methods Potential Piffalls

\section{Shift change:}

- Having key staff physically present for verbal report to next shiff

- Reviewing previous shift notes with all staff on a unit before starting next shift

- Providing staff starting a new shift or returning from leave of absence with sufficient time to review resident updates

Ad hoc processes:

- Group "huddles" between or during shifts that include all staff roles, to review key information. This rarely requires more than ten minutes but is recognized as being very valuable.
- Staff continuity was recognized as beneficial: need to anticipate that new or part-time / occasional staff, or staff returning from absences, require orientation to protocols specific to unfamiliar units and to the residents.

- Staff working shorter shifts have less time for communication and orientation.

- Resident coverage: While involving as many staff as possible in team meetings was generally seen as beneficial, concerns were raised about having adequate staff available to cover the units during meetings. on an already busy daily schedule. The minimum standard in Ontario LTC homes is for weighings to be done monthly, usually during the day shift (Ministry of Health and Long-Term Care [MOHLTC], 2007). Weights are recorded, in writing or electronically, in the resident chart. Some facilities were reported as being able to obtain resident weights twice a week during bath time, also mandated to occur at this frequency in Ontario (MOHLTC, 2007). While bath times for individuals are performed at consistent times, they do not necessarily occur in the morning. However, for residents who require significant assistance with transfers, weighing at bath time was deemed to be more practical.

Workshop participants generally accepted that residents with HF often require more frequent weight measurements. Some facilities were described as being able to weigh a small number of residents with HF more often, perhaps three times a week to daily, but for limited periods of time, given the significant burden placed on PSWs. The importance of educating PSWs about the rationale for regular weighings was repeatedly emphasized. PSWs who attended the workshops, and who were educated regarding the rationale for frequent weighing, were accepting of the need to carry out this task, particularly for residents with unstable HF. However, they emphasized the need to consider reducing the frequency of weighing as residents stabilize.

Frequent weighing of LTC residents with HF can only be meaningful if measurements can be compared to a target weight, in order to correctly ascertain changes in volume status. Concerns were raised that recording more frequent measurements might be limited by the type of electronic medical record software in use in a specific home, complicating the identification of important changes. Some software solutions allow for automatic alerts to be set, although participants pointed out that these often result in a number of falsepositive alerts. Furthermore, staff need to access computers regularly in order to be notified of such alerts.
There was general consensus that tracking frequent weights using paper flow sheets was likely to be the most efficient way of detecting changes in weight. Further, it was suggested that PSWs require specific guidelines for reporting significant changes in weight to RPNs or RNs. Finally, participants identified considerations related to the measurement process (Table 7).

\section{Educational Interventions and Needs}

Deficits in knowledge about all aspects of HF and among all staff were documented in all phases of this project (Heckman, Boscart, McKelvie, et al., 2014; Kaasalainen et al., 2013; Marcella et al., 2012; Newhouse et al., 2012; Strachan et al., 2014). Workshop participants generally agreed that all staff require HF education that is tailored to their level of training. In particular, emphasis is required about the importance of nonspecific changes in function and cognition as early markers of disease exacerbation. Education of frontline staff, including PSWs and RPNs, should be closely linked to strategies to improve staff confidence and interprofessional communication skills. Residents and family caregivers also require information about HF, its complications and unpredictable course, along with various treatments, including the importance of dietary measures. Workshop participants agreed that, although reading material and electronic media may play a role in patient education, LTC staff should also be taught to provide this information.

Workshop discussants agreed that effective staff education requires a multifaceted, interactive strategy. The notion of having specific HF champions at each home was met with mixed feelings. Whereas some discussants felt that this was an appropriate strategy, others pointed out that the notion of "champions" is overused in LTC, vulnerable to personnel changes, and therefore less effective than a strategy with a broader reach across all staff levels. Specific staff-specific educational needs were discussed and are summarized in Table 8. 
Table 7: Considerations for accurate weight measurement in LTC

\begin{tabular}{ll}
\hline Domain & Considerations \\
\hline Device specific & - Need for quality devices \\
& - Access to consistent device for each resident \\
Resident specific & - Need to "zero" scales before use \\
& - Lift scales considered less accurate \\
& - Ensure continence garments are dry \\
& - Ensure consistent state of dress/undress \\
& - Consistent time of day (preferably before breakfast) \\
\hline
\end{tabular}

Appropriate pharmacotherapy for LTC residents with HF: Most physicians participating in the workshops expressed an understanding of the importance of first-line therapies for $\mathrm{HF}$, specifically angiotensin converting enzyme inhibitors (ACEi), beta-blockers, and mineralocorticoid receptor antagonists (MRA), as well as other HF therapies. They also expressed concerns regarding their use, including the potential risks (hypotension, renal insufficiency and electrolyte abnormalities) and how these might be mitigated or prevented. Workshop participants observed that important goals of therapy among LTC residents include maintaining quality of life and function, and avoiding hospitalization; reiterating the role of first-line therapies in achieving these outcomes is an important consideration to encourage LTC physicians to prescribe the aforementioned medications.

Several participants noted that some LTC homes have access to an emergency medication kit to manage acute illnesses such as HF. These kits include injectable furosemide (for intramuscular administration), morphine, and midazolam. Use of these medications does require authorization by the LTC physician, but several discussants indicated that these kits facilitate the care of residents with acute HF in the LTC home, thus avoiding acute care transfers.

Some LTC homes have reliable access to a pharmacy consultant who conducts quarterly medication reviews. Other homes may have access to occasional pharmacy students. Pharmacists can provide support to physicians regarding the usage of HF medications, provided that they have a reasonable knowledge of their usage in frail HF patients, and are often available by phone.

The notion of flexible diuretic regimens, commonly used in outpatient HF management clinics and in which a daily diuretic dose is adjusted according to a patient's most recent weight in relation to a target weight, was met favourably by some LTC physicians and with reservations by others. Some physicians were reluctant to consider authorizing changes in diuretic doses, based on a reported weight, without having clinically assessed the

Table 8: Specific considerations regarding educational needs of LTC staff

\begin{tabular}{|c|c|}
\hline Need & Considerations \\
\hline Physician needs & $\begin{array}{l}\text { - Assess volume status and determine target weights for residents } \\
\text { - Prescribe flexible diuretic regimens } \\
\text { - Monitor vital signs (including orthostatic vitals), renal function, and electrolytes } \\
\text { - Understand how to prescribe evidence-based HF medications } \\
\text { - Manage and anticipate side effects of HF therapy, including managing hypotension, } \\
\text { bradycardia, and renal insufficiency }\end{array}$ \\
\hline
\end{tabular}

HF $=$ heart failure 
resident. Such policies would require strict parameters for which a clinical assessment would be required, the use of reliable scales and weighing procedures, and would have to be approved by the Medical Advisory Committee for each home.

Advance care planning: Communication among staff, residents, and family caregivers: Effective communication with residents and families was seen as particularly important by workshop participants when establishing and following advance care plans. Usually, these wishes are addressed when a resident is admitted to LTC, and revisited annually. These important discussions are often lengthy and require staff to invest significant amounts of time to prepare, engage with residents and families, and follow up. Annual mortality rates from HF in LTC may be as high as 50 per cent (Heckman, Boscart, \& McKelvie, 2014). HF exacerbations are also common, often leading to transfers to acute care. Workshop participants reported that despite advance directives against such transfers, family members frequently overturn these directives in emergency situations. As a result, LTC staff may feel frustrated that resident wishes are not respected, and families may experience regret for overturning advance directives. Often, LTC residents are considered to have poor experiences in the acute care setting, such that managing acute problems in the LTC home is generally preferred to transferring them to acute care (Marcella et al., 2012). Reasons underlying decisions to overturn advanced directives, although not fully explored during the workshops, were felt to reflect a combination of factors including poor communication between LTC staff and families, staff unfamiliarity with the course and prognosis of advanced HF, and perceptions by some families that LTC staff lack confidence in managing HF exacerbations in the home. Workshop participants agreed that advance directives ought to be discussed more frequently, particularly for residents with frequent exacerbations, fluctuating course, or progressive decline in overall health, and that these discussions would be facilitated by stronger staff knowledge about the trajectory of HF.

Effective communication with families was noted by workshop participants as challenging. Often, LTC staff forget that many cognitively impaired residents remain able to provide meaningful input into their own care. Participants agreed that engaging residents and family caregivers as members of the care team, and encouraging frequent and open two-way communication has a favourable impact on the implementation of advance care plans. However, participants also noted that the quality of interprofessional communication among LTC staff impacts the quality of communication with residents and families. Much communication with family was done by phone, primarily by nurses, with physicians calling less frequently. Information exchanged with families was often poorly communicated to incoming staff at shift change. This outcome has a negative impact on family confidence with the care team.

A particular concern raised by workshop participants related to residents with multiple family caregivers who communicate poorly among themselves. This can result in multiple requests to different LTC staff at different times, leading to greater staff workload and frustration, concerns over resident confidentiality, and increasing the risk that conflicting information is shared with the family. LTC staff face the challenge of having to identify a lead-contact family member with whom to share treatment information or HF education, and encourage this person to inform other family members. PSWs bear the brunt of this problem, as they represent the most familiar caregiver to the family, despite being provided with the least amount of information about resident health from other staff, and thus not being well equipped to answer questions. Thus, PSWs are sometimes compelled to pass family health concerns on to the RPNs, who may then notify RNs and physicians. As this "communication chain" lengthens, the risk of information being lost or distorted increases, often leading to further frustration and mistrust by family members. These interprofessional factors, leading to mistrust of LTC staff by families, were also felt to contribute to the overturning of advance care plans.

\section{Interventions Geared to the System Level and the Broader Community}

Communication with acute care: Communication between LTC and acute care facilities was identified by workshop participants as generally problematic. Although some information (at times, often in illegible handwriting) is sent back with the resident, very little diagnostic and management information is shared with LTC homes when a hospitalized resident is admitted to, or discharged from, acute care. This is particularly true of residents seen in emergency departments and discharged back to LTC without being hospitalized. At times, only a prescription is sent back with the resident. On some occasions, diagnostic results and clinical notes are sent to the LTC physician's private office rather than to the LTC home, while on other occasions these are sent to the resident's previous physician. Participants pointed out that acute care hospitals often cite "patient confidentiality" as a reason for not sharing such information, yet the LTC home is within the defined "circle of care" for hospitalized residents. Consequently, some LTC homes are obliged to expend significant effort contacting emergency and medical records departments in order to obtain relevant medical information. In addition, although many LTC homes share health information, including medical directives, progress notes, and diagnostic and medication lists, 
when a resident is transferred to acute care, the general perception is that this information is usually ignored. Finally, some residents are discharged back to LTC with the expectation that certain treatments or investigations be carried out that are beyond LTC staff or facility capabilities. Several measures to improve communication between LTC, acute care, and other health service providers, are as follows:

- Employ nurse practitioners, who are generally funded through regional health authorities, to conduct urgent assessments and facilitate resident transitions.

- Mandate protocols for direct communication between acute care and LTC before a resident is accepted back to LTC. For example, some workshop participants had access to a service whereby a designated contact person in acute care communicates with LTC homes regarding hospitalized residents; this service was found to be beneficial despite restriction to daytime hours on weekdays.

- Link LTC electronic medical records with those of regional providers.

- Educate LTC and acute care staff regarding privacy regulations and how to more effectively apply these when dealing with resident transfers.

- Improve access to diagnostic testing and specialists by means of the following:

- Enhance access to laboratory testing, which may be limited to once weekly or, if needed more often, associated with additional costs to some LTC homes;

- Ensure more timely availability of reports to support LTC clinicians who need to make urgent clinical decisions;

- Increase access to specialists (e.g., geriatricians, internists, cardiologists), either directly or through a specialist hotline for rapid phone consultation.

Developing facility-wide strategies and protocols for dietary interventions: Malnutrition was regarded by workshop discussants as an important concern in LTC. Unpalatable food contributes to malnutrition. Although salt reduction may be desirable with respect to HF, it was recognized that sodium reduction can reduce the palatability of food, thus leading to other health challenges. Workshop participants discussed two potential approaches to reducing sodium: the first focused on the snacking and dietary habits of individual residents; the second addressed dietary sodium at the institutional level. The former approach was seen as requiring education of residents and their families by individual staff and dietitians, whereas the latter was seen as requiring the involvement of facility food services.

The availability of dietitians was often limited to one or two days a week. Dietitians generally reviewed residents every three months, and in greater depth if residents experience significant changes in weight. Dietitians were not considered to be in a position to monitor resident weights more frequently, as might be required to manage HF. Finally, although dietitians were able to assist residents and families develop a dietary plan, they had little control over daily choices, which may not be heart healthy. Assessing dietary intake of nutrients could be complicated. Often, the proportion of meals eaten was recorded, but actual intake was difficult to interpret given that absolute portion sizes were not recorded.

Although some facilities prepared fresh food on site and provide low sodium options, others relied on prepackaged food, which is generally higher in sodium content but less costly. One dietician attending a workshop suggested that, on a "good day", the average sodium intake of residents in the home she served was well over 4 grams per day, which was markedly higher than what HF guidelines recommend. In addition, residents who required pureed diets were at a further disadvantage, as these diets required the addition of moisture, generally in the form of salty gravy.

Developing facility-wide strategies to increase physical activity levels of residents: The importance of physical activity in persons with HF is well established, and was widely accepted by workshop participants. However, the optimal manner in which to deliver exercise in LTC has not been established and is subject to significant resource constraints. When considering the delivery of exercise to LTC residents, participants identified several important considerations to enhance the level of physical activity among LTC residents with HF (Table 9).

\section{Recommendations for HF Management in LTC}

Based on analysis and review of the information collected in the workshops, the study investigators developed 17 recommendations, as well as practical tips on implementation.

\section{Interprofessional Communication}

Recommendation 1: Given that optimal HF management in LTC is contingent on effective, mutually respectful and trusting communication, all staff should receive education and training in communication skills with a focus on interprofessional collaboration.

Recommendation 2: Given that shift change is of critical importance to ensure that important information regarding potentially unstable residents be effectively shared, LTC homes should develop shift-change communication protocols that combine written and verbal strategies.

Recommendation 3: Given that each member of the care team can make important contributions to the wellbeing of LTC residents, all staff roles, including PSWs, should be actively involved in discussions at shift change, 
Table 9: Considerations to promote physical activity among LTC residents with HF

\begin{tabular}{|c|c|}
\hline Principles & Considerations \\
\hline Families often overestimate resident abilities & $\begin{array}{l}\text { - Staff training and resources are required to evaluate how much activity } \\
\text { residents can participate in, and how to effectively share this information with } \\
\text { family caregivers and residents. }\end{array}$ \\
\hline
\end{tabular}

LTC $=$ long-term care

physician rounds, new admissions, or on an ad hoc basis when resident health concerns are identified.

Recommendation 4: Given the importance of advance care planning in LTC in general and for residents with HF specifically, advance care planning should be regularly discussed with all LTC residents and their family caregivers.

Recommendation 5: Given the importance of regular weighings in the early identification of HF decompensation, residents with HF should be weighed at least twice a week, or more often when unwell, and under conditions that are as consistent as possible.

Recommendation 6: Given the importance of accurate weighings to monitor residents with HF, LTC homes should implement formal protocols to standardize the measurement of weight for LTC residents.

A recent report from the Institute of Medicine emphasizes several key aspects of effective interprofessional education (Institute of Medicine, 2015). It is essential to deliver such education in a structured manner that engages all providers and promotes collaborative learning within the workplace itself. Aligning the education with health care delivery allows for greater patient engagement, and a closed feedback loop for learners to appreciate their impact on the patient. The educational intervention must align with organizational and individual priorities for quality improvement. Our previous work also emphasized the importance of multimodel learning activities, including bedside teaching (Heckman, Boscart, McKelvie, et al., 2014). Table 10 summarizes key practical tips emerging from the workshops regarding the implementation of interprofessional communication processes.

\section{Educational Interventions}

Recommendation 7: Given the importance of HF as a clinical entity in LTC, and given the existence of significant knowledge gaps among all staff roles, and among residents with HF and their family caregivers, all LTC staff should receive HF-related educational intervention tailored to their level of professional education. HF-related education should also be provided to residents and family caregivers.

Recommendation 8: Given the importance of first-line pharmacotherapies in the management of HF, particularly with respect to improving symptoms in residents with reduced left ventricular function, LTC homes should have protocols available to assist physicians in optimizing the appropriate use of first-line HF therapies (ACEi, beta blockers, MRAs).

Recommendation 9: Given the importance of other HF pharmacotherapies in the management of HF, LTC homes should have protocols available to assist physicians optimize the use of other HF therapies such as = angiotensin receptor blockers, digoxin, vasodilators, and diuretics.

Recommendation 10: Given that HF exacerbations may occur suddenly during times when LTC staffing 
Table 10: Practical tips related to interprofessional communication

\begin{tabular}{|c|c|}
\hline Domains & Tips \\
\hline
\end{tabular}

is short, such as nighttime, LTC staff should be trained to use readily accessible emergency medication kits containing oral and parenteral medications, including furosemide, morphine, and benzodiazepines, in order to manage symptoms of acute HF and avert transfer to acute care facilities.

Recommendation 11: Given that many LTC residents with HF also suffer from significant co-morbidities requiring the prescription of multiple drugs, LTC physicians should receive training on optimal prescribing for complex older residents with HF.

Recommendation 12: Given that many LTC residents with advanced HF experience frequent fluctuations in fluid retention and weight, LTC staff should be trained on how to implement protocols for flexible diuretic regimens to be used for residents with HF whose weights fluctuate over the course of several days and in whom a firm target weight has been determined.

Most of the care to residents of LTC homes is provided by PSWs and RPNs, and thus most of the opportunities for clinical observation, either of early signs and symptoms of illness, or to monitor the effects of a treatment, must involve these staff members. Recognition of impending HF requires awareness that its manifestations can be non-specific, and observed at different times of the day. In keeping with the recommendations on interprofessional education by the Institute of Medicine, it is thus essential that this education also be delivered in a workplace setting (Institute of Medicine, 2015). Tables 8 and 11 provide additional details of educational needs of LTC stakeholders.

\section{Communication with Acute Care and Other Community Providers}

Recommendation 13: Given the importance of ensuring the timely and comprehensive exchange of information between LTC homes and acute care hospitals, LTC administrators should establish, with their counterparts in acute care hospitals, protocols to facilitate the communication of health information specific to residents of LTC who require transfer to or from an acute care hospital.

Recommendation 14: Given the importance of ensuring the timely and comprehensive exchange of information between LTC homes and placement agencies, LTC administrators should establish, with their counterparts in community placement agencies, protocols to facilitate the communication of health information when residents are newly admitted to LTC.

\section{Recommendations Regarding Nutritional Interventions and Physical Activity}

Recommendation 15: Given that sodium restriction is recommended as an important modality in the management of chronic HF, a dietitian should review the overall sodium content of foods available and served at an LTC facility in order to promote lower sodium choices 
Table 11: Practical tips related to staff educational needs

\begin{tabular}{ll}
\hline Domain & Tips for Meeting that Need \\
\hline HF education & - Multimodal, interprofessional interventions are preferred \\
Appropriate pharmacotherapy & Prescribed protocols should outline in a clear manner how to: \\
& -monitor residents receiving HF medications; \\
& -manage hypotension, dizziness, bradycardia, or renal dysfunction; and \\
& -use alternative therapies in the case of true intolerance to a first-line medication (e.g., ARBs \\
& for residents intolerant of ACEi) \\
- & Engagement of consultant pharmacists to assist LTC physicians may be helpful \\
- & Clinicians involved in advance care planning discussions must understand the unpredictable \\
& course and prognosis of HF prognosis in order to be able to confidently address resident \\
- & and family caregiver concerns. \\
- Advance care plans should be reviewed annually and when clinically important events & occur, such as HF exacerbations, clinically significant deterioration in the resident's \\
& condition, or when requested by the resident or family caregivers. \\
- A lead family member should be identified as substitute decision-maker (SDM), this & information should be shared with other family members.
\end{tabular}

$\mathrm{ACE}=$ angiotensin-converting-enzyme inhibitor; $\mathrm{ARBs}=$ angiotensin receptor blockers; $\mathrm{HF}=$ heart failure

and achieve facility-wide reductions in dietary sodium intake.

Recommendation 16: Although water restriction is recommended for persons with HF and severe fluid volume overload resistant to diuretics or persistent hyponatremia, but considering the extensive burden of co-morbidities and frailty among LTC residents with $\mathrm{HF}$ and who are thus likely to be nearing the end of life, and also considering the suffering that can result from excessive thirst, routine water restriction for severely ill LTC residents is not recommended.

Recommendation 17: Given the importance of physical activity to help maintain function and quality of life in LTC residents, including those with HF, LTC facilities should develop, in consultation with all staff and with residents and family caregivers, processes to promote and integrate physical and recreational activities into all aspects of daily life and care.

Tables 9 and 12 provide tips on implementing these recommendations.

\section{Discussion}

This article describes the results of interprofessional workshops carried out in LTC homes with the goal of developing pragmatic recommendations on how to implement HF care processes in LTC. This work builds upon prior consultation phases to identify barriers and potential solutions regarding the implementation of the Canadian Cardiovascular Society (CCS) HF guidelines in LTC homes (Heckman, Boscart, McKelvie, et al., 2014; Kaasalainen et al., 2013; Marcella et al., 2012; Newhouse et al., 2012; Strachan et al., 2014). These recommendations focus on improving interprofessional communication among LTC stakeholders, providing
HF-related education geared towards the needs of each LTC staff role, engaging residents and their family caregivers in effective advance care planning discussions, improving communication with community health service providers, and developing institutional policies related to nutritional sodium intake and physical activity. Engagement of all LTC staff, and especially PSWs, is an essential element of these recommendations.

HF is a common and important co-morbidity among LTC residents, in whom it is associated with significant morbidity and health service utilization. Achieving optimal HF outcomes in this population is complicated by the clinical complexity of LTC residents, who are often frail and affected by multiple co-morbidities and geriatric syndromes, notably falls and cognitive impairment (Harkness, Heckman, \& McKelvie, 2012). Despite evidence suggesting that frail seniors with HF also benefit from the therapies recommended in guidelines, the care provided to this population often fails to reflect these recommendations (Heckman, Boscart, \& McKelvie, 2014). HF management programs, based on the chronic disease management and prevention model, have been shown to improve outcomes among frail seniors living in the community (Hunt et al., 2009; McKelvie et al., 2013; McMurray et al., 2012). Although a small number of studies of HF management programs in LTC have shown the potential to reduce health service utilization, these programs have either targeted a relatively well subset of LTC residents or benefit from resources generally unavailable to typical LTC homes. Moreover, none of these programs were developed with the systematic stakeholder consultation required to maximize the chances for sustainability (Boxer et al., 2012; Hancock et al., 2012; Jacobs, 2011; Martinen \& Freundl, 2004; Tjam et al., 2006; Valle et al., 2001). 
Table 12: Practical tips related to nutritional recommendations

\begin{tabular}{ll}
\hline Nutritional Recommendations & Tips for Meeting that Recommendation \\
\hline Dietary interventions & - Flavourful moisture alternatives to high-sodium gravy for pureed foods; \\
- Consideration of removal of salt shakers from tables frequented by residents with HF; & - Availability of salt alternatives, avoiding products with high potassium content; and \\
- Education of families and PSWs, who generally have a more accurate sense of what residents & are eating, to support informed nutritional choices, including snacks. \\
- The risks and benefits of water restriction should be discussed with the residents and/or SDM, & as long as this discussion is contextualized to the residents overall state of health, and their \\
& stated goals of care.
\end{tabular}

HF = heart failure; PSWs = personal support workers; SDM = substitute decision-maker

The recommendations described herein are based on a three-phase and iterative process centred on extensive consultation with LTC stakeholders, including physicians, RNs, RPNs, PSWs, as well as residents and family caregivers. These consultations provided rich and in-depth data regarding barriers to HF management in LTC, and identified key elements of an intervention to address these barriers. These data were used to develop 17 recommendations to guide the development of HF care processes in LTC. An expert panel oversaw all three phases of the project and reviewed the final recommendations to ensure that they remained true to the CCS HF guidelines and respected the scopes of practice of the various LTC staff roles. Most importantly, unlike previous HF management programs in LTC, the process leading to the formulation of these recommendations was anchored in ongoing and active stakeholder engagement, which is essential in order to maximize the likelihood of successful and sustainable implementation (Colón-Emeric et al., 2007; Grimshaw et al., 2004; Lewis et al., 2005; Mentes \& Tripp-Reimer, 2002; Mutasingwa, Ge, \& Upshur, 2011).

Limitations of this work should be acknowledged. A limited number of LTC homes were involved in the focus groups and workshops, which was a consequence of the consultation methods we utilized. However, these homes were deliberately selected to provide variability in terms of setting (urban, rural, and northern) and ownership (private, for-profit, and not-for-profit). Furthermore, Phase 1 of the study did include a province-wide survey. The use of multiple data sources and consultation methods (survey, focus groups, interviews, workshops, panels) does provide reassurance with respect to the validity of the recommendations through methodological triangulation (Creswell, 2009). Finally, a number of system and resource issues, such as availability of portable diagnostics such as echocardiography, were not addressed. However, interprofessional literature suggests that when care providers develop disease management interventions, it is essential that they first address factors closest to the patient (Newhouse et al., 2012). In this instance, there was overwhelming consensus on the need to address interprofessional communication and LTC staff education. Nevertheless, the validity of the recommendations should be evaluated in other LTC settings, both within and beyond Ontario.

The findings from these LTC stakeholder engagement workshops, and the ensuing recommendations and practical tips, provide a basis for further stakeholder engagement to specifically develop, implement, and evaluate HF care processes in LTC. Preliminary findings of a pilot intervention in two LTC homes suggest that a multimodal interprofessional and HF education intervention based on this work is effective and has the potential to improve resident outcomes (Brohman et al., 2015; Huson et al., 2015).

\section{Conclusion}

Through an iterative process relying heavily on stakeholder engagement and expert panel consultation, we have developed practical recommendations on how to implement care processes to manage HF in LTC. The formulation of these recommendations is an important first step towards developing effective and sustainable HF management programs for LTC.

\section{References}

Ambrosy, A. P., Fonarow, G. C., Butler, J., Chioncel, O., Greene, S. J., Vaduganathan, M., et al. (2014). The global health and economic burden of hospitalizations for heart failure: Lessons learned from hospitalized heart failure registries. Journal of the American College of Cardiology, 63, 1123-1133.

Bleumink, G. S., Knetsch, A. M., Sturkenboom, M. C. J. M., Straus, S. M., Hofman, A., Deckers, J. W., et al. (2004). Quantifying the heart failure epidemic: Prevalence, incidence rate, lifetime risk and prognosis of heart failure. European Heart Journal, 25, 1614-1619.

Boxer, R. S., Dolansky, M. A., Frantz, M. A., Prosser, R., Hitch, J. A., \& Piña, I. L. (2012). The Bridge Project: Improving heart failure care in skilled nursing facilities. Journal of the American Medical Directors Association, 13, 83e1-83e7. 
Brohman, L., Franco, L., Huson, K., Boscart, V., McKelvie, R., Hirdes, J. P., et al. (2015). Core heart teams: Improving interprofessional communication for heart failure management in long-term care. Canadian Journal of Cardiology, 31, s274.

Colón-Emeric, C. S., Lekan, D., Utley-Smith, Q., Ammarell, N., Bailey, D., Corazzini, K., et al. (2007). Barriers to and facilitators of clinical practice guideline use in nursing homes. Journal of the American Geriatrics Society, 55, 1404-1409.

Creswell, J. (2009). Research design: qualitative, quantitative and mixed method approaches (3rd ed.). Thousand Oaks, CA: Sage.

Go, A. S., Mozaffarian, D., Roger, V. L., Benjamin, E. J., Berry, J. D., Blaha, M. J., et al. (2014). Heart disease and stroke statistics-2014 update: A report from the American Heart Association. Circulation, 129, e28-e292.

Grimshaw, J. M., Thomas, R. E., MacLennan, G., Fraser, C., Ramsay, C. R., Vale, L., et al. (2004). Effectiveness and efficiency of guideline dissemination and implementation strategies. Health Technology Assessment, 8, 1-84.

Gruneir, A., Bell, C. M., Bronskill, S. E., Schull, M., Anderson, G. M., \& Rochon, P. A. (2010). Frequency and pattern of emergency department visits by long-term care residents-A population-based study. Journal of American Geriatrics Society, 58, 510-517.

Hancock, H. C., Close, H., Mason, J. M., Murphy, J. J., Fuat, A., de Belder, M., et al. (2012). Feasibility of evidence-based diagnosis and management of heart failure in older people in care: A pilot randomized controlled trial. BMC Geriatrics, 12, 70.

Harkness, K., Heckman, G. A., \& McKelvie, R. S. (2012). The older patient with heart failure: High risk for frailty and cognitive impairment. Expert Reviews Cardiovascular Therapy, 10, 779-795.

Heckman, G. A., Boscart, V. M., \& McKelvie, R. S. (2014). Management considerations in the care of elderly heart failure patients in long-term care facilities. Future Cardiology, 10, 563-577.

Heckman, G. A., Boscart, V. M., McKelvie, R. S., D'Elia, T., Osman, O., Kaasalainen, S., et al. (2014). Perspectives of primary-care providers on heart failure in long-term care homes. Canadian Journal on Aging, 33, 320-335.

Heckman, G. A., Tannenbaum, C., Costa, A. P., Harkness, K., \& McKelvie, R. S. (2014). The journey of the frail older adult with heart failure: Implications for management and health care systems. Reviews in Clinical Gerontology, 24, 269-289.

Hirdes, J. P., Mitchell, L., Maxwell, C. J., \& White, N. (2011). Beyond the 'iron lungs of gerontology': Using evidence to shape the future of nursing homes in Canada. Canadian Journal on Aging, 30, 371-390.

Hunt, S. A., Abraham, W. T., Chin, M. H., Feldman, A. M., Francis, G. S., Ganiats, T. G., et al. (2009). Focused update incorporated into the ACC/AHA 2005 Guidelines for the Diagnosis and Management of Heart Failure in Adults: A report of the American College of Cardiology Foundation/American Heart Association Task Force on practice guidelines: Developed in collaboration with the International Society for Heart and Lung Transplantation. Circulation, 119, e391-e479.

Huson, K., Boscart, V., McKelvie, R., Hirdes, J.P., Stolee, P., \& Heckman, G. (2015). Interprofessional care processes to manage residents with heart failure in long-term care. Canadian Journal of Cardiology, 31, S128.

Institute of Medicine. (2015). Measuring the impact of interprofessional education on collaborative practice and patient outcomes. Washington, DC: The National Academies Press.

Jacobs, B. (2011). Reducing heart failure hospital readmissions from skilled nursing facilities. Professional Case Management, 16, 18-24.

Kaasalainen, S., Strachan, P. H., Heckman, G. A., D’Elia, T., McKelvie, R. S., McAiney, C., et al. (2013). Living and dying with heart failure in long-term care: Experiences of residents and their family members. International Journal of Palliative Nursing, 19, 375-382.

Lewis, D. L., Jewell, D., Turpie, I., Patterson, C., McCoy, B., Baxter, J. (2005). Translating evidence into practice: The case of dementia guidelines in specialized geriatric services. Canadian Journal on Aging, 24, 251-260.

Marcella, J., Nadarajah, J., Kelley, M., Heckman, G., Kaasalainen, S., Strachan, P., et al. (2012). Understanding organizational context and heart failure management in long term care homes in Ontario, Canada. Health, 4, 725-734.

Martinen, M., \& Freundl, M. (2004). Managing congestive heart failure in long-term care. Journal of Gerontological Nursing, 30, 5-12.

McKelvie, R. S., Moe, G. W., Ezekowitz, J. A., Heckman, G. A., Costigan, J., Ducharme, A., et al. (2013). The 2012 Canadian Cardiovascular Society heart failure management guidelines update: Focus on acute and chronic heart failure. Canadian Journal of Cardiology, 29, 168-181.

McMurray, J. J., Adamopoulos, S., Anker, S. D., Auricchio, A., Böhm, M., Dickstein, K., et al. (2012). ESC Guidelines for the diagnosis and treatment of acute and chronic heart failure 2012. European Journal of Heart Failure, 14, 803-869.

Mentes, J. C., \& Tripp-Reimer, T. (2002). Barriers and facilitators in nursing home intervention research. Western Journal of Nursing Research, 24, 918-936.

Ministry of Health and Long-Term Care [MOHLTC]. (2007). Long-Term Care Homes Act, 2007 (LTCHA) O. Reg. 79/10 Proposed amendments to Ontario Regulation 79/10 under the Long-Term Care Homes Act. Retrieved from the Ontario Ministry of Health and Long-Term Care website: http:/ / www.health.gov.on.ca/en/public/programs/ltc/docs/ ltcha_guide_phase1.pdf 
Mutasingwa, D. R., Ge, H., \& Upshur, R. E. G. (2011). How applicable are clinical practice guidelines to elderly patients with comorbidities? Canadian Family Physician, 57, e253-e262.

Newhouse, I. J., Heckman, G., Harrison, D., D'Elia, T., Kaasalainen, S., Strachan, P. H., et al. (2012). Barriers to the management of heart failure in Ontario longterm care homes: An interprofessional care perspective. Journal of Research in Interprofessional Practice and Education, 2, 278-295.

Strachan, P. H., Kaasalainen, S., Horton, A., Jarman, H., D'Elia, T., Van Der Horst, M., et al. (2014). Managing heart failure in the long-term care setting: Nurses' experiences in Ontario, Canada. Nursing Research, 63, 357-365.

Tjam, E. Y., Pletz, D. E., Hirdes, J. P., Smith, S. J., Costigan, J., Wilwerth, B., et al. (2006). Prevention of avoidable acute care use by retirement and long term care residents: Management of chronic heart failure through the use of an innovative inter-disciplinary education program for care providers (EPOCH study). Retrieved from the Pulsus website: http://www.pulsus.com/ccc2006/abs/056.htm

Valle, R., Chinellato, M., Milani, L., \& di Piave, S. D. (2001). Impact of a guideline-based management on outcomes of very old persons with heart failure living in nursing homes. Archives of Internal Medicine, 161, 2264-2265. 